\title{
Finite element modelling for fruit stress analysis - a review
}

\begin{abstract}
Background: A deep understanding of the motion and the intensities of forces endured by the fruits overtime during the various handling processes is necessary if improvements are to be made in the handling systems or related tools. The finite element method (FEM) has been used to investigate the effect of mechanical loading on fruit before developing a prototype of the intended design. Simulation tests were performed to visualise the time-dependent deformation behaviour. Various studies have been reported on the use of FE for studying the effect of compression and drop forces on fruits. However, to the best of the knowledge of the authors, the application of finite-element analysis in fruit mechanics has not been summarized yet. Scope and approach: This review is concerned with establishing the introductory concept of FEM for evaluating fruit response under the static and dynamic loading. The FE equations were formulated based on the stress-strain constitutive equations. Key findings and conclusions: The review summarises the application of FEM for fruit stress-strain analysis. A brief description of the fundamentals and the existing models that have been developed to cope with problems of fruit mechanics are reported. This paper provides a review of the main FEM studies and a comparison between different types of stress analysis as well as outlining the potential use of FEM for future reference. This review can become an appropriate, timely and beneficial reference for any relevant follow-up research for the assessment of the quality of agricultural produces.
\end{abstract}

Keyword: Finite element method; Fruit response; Stress-strain analysis 